\title{
Content Based Image Retrieval for Community Retrieval from Given Nationality using an Efficient Combination Algorithm
}

\author{
Shaik. Rahamtula, T. Jaya
}

\begin{abstract}
There is tremendous requirement of such technique which can fulfill the entire requirement for retrieval of an image from available dataset which comes under computer vision. In this paper we discussed about the one of the application of CBIR using an efficient combination of two techniques. The application is retrieval of people images from database that comes under minority. In this paper we used an efficient combination of color image histogram technique and edge orientation histogram technique by dividing original image into small subblocks. The feature vector is formed by combination of two features obtained by above methodologies. The final features obtained by query image will be compared with the feature vector of database images using a new Canberra Distance classifier. Proposed method is designed for multiple self-prepared and some collected from internet databases. Our method includes the efficient integration of features such as color, texture, shape and orientation. The proposed method is compared with state of art techniques to prove the stable and highest accuracy of proposed work.
\end{abstract}

Keyword: Integration of Features, Color Histogram, Edge Orientation Histogram, Canberra Distance, Content Based

\section{INTRODUCTION}

The minority costume is the primary or basic picture of the ethnic association differentiating the proof. For the present, the minority costumes are uniquely covered by method for historical centers statically. CBIR is an extremely fundamental inside the subject for test acknowledgment and man-made reasoning. In spite of the fact that nationwide minority apparel picture have confused visual capacities, the guideline characteristics by and by are attire shading, fabric surface and totem frame, which are as per the picture include in PC inventive and perceptive. So we can utilize regular component extraction calculations to extricate the abilities of minority costumes image. At presentation, an enormous number of techniques on extraction of shading, surface and frame capacities had been advanced and have just acquired reasonable impacts in number of fields. Shading is the most predominant and recognizing unmistakable trademark. The current shading highlight extraction systems incorporate shade histogram [1], shading minute [2], SCV [3] and SC [4].

FCD, SD have acknowledged comprehensive of wide assortment of HD [5]. Surface utilized to determine unpleasantness. Numerous specialists prescribe different calculations for the GLCM [6], LBP [7], LDP [8], etc.

Revised Manuscript Received on October 15, 2019.

Shaik. Rahamtula, Research scholar, Department of Electronics and Communication Engineering, VELS Institute of Science Technology and Advanced Studies, Pallavaram, Chennai, Tamilnadu, India.

Dr. T. Jaya, Assistant Professor, Department of Electronics and Communication Engineering, VELS Institute of Science Technology and Advanced Studies, Pallavaram, Chennai, Tamilnadu, India. Image Retrieval.

Ordinary frame descriptors indicate second invariants, FRC [9] and HOG [10]. For photograph recovery, Guang-Hai Liu proposed one technique that is nothing but multi-texton histogram. It incorporates the usage of co-event framework and histogram by method for speaking to the trait of coevent network the utilization of histogram. It successfully incorporates shade, surface, frame and shading design data as photograph recovery. Perspective of many picture work extraction calculations dependent on multi-capacities have been practically connected in picture recovery, this paper displays a complete component descriptor to explicit the affluent noticeable abilities gave in minority dress picture. Histogram adjustment plays indispensable capacity in numerous virtual handling bundles and besides while the imperative in a virtual photograph is on the nearby assessment esteems by utilizing histogram evening out system we can expand the levels of world assessment of numerous selective pix which has the fundamental information at the nearby appraisal esteems.

\section{LITERATURE REVIEW}

CBIR (Content Based Image Retrieval) has become lengthy sooner than 1990 and practically no papers have been posted at that point, but the range of papers posted in view that 1997 is growing. Here, we know different type of CBIR algorithms, because it gives different results. For that research results we get many layers of responsibilities in many algorithms. These many layers of responsibilities for image extracting the multidimensional capabilities of an image will perform with an image in that database then we observe the different database with an image. We have to improve the database with extracted information from the indexed and snap shots as an improved image as a retrieval image. To improve the image quality we have to consist the below parameters i.e.., shape, shade, texture and rest of the image fusion. CBIR has been established in 1990 and after that there posted different scope of papers developed in1997.There are different CBIR calculations. These calculations main thought is to take an image into a few layers of obligations in CBIR. Those layers main duties will envelop by removing the three dimensional capabilities of a image and take another image from inside of the database, then will perform the machine database with image. These database with extracted data from duplications and it will be affected the normal execution from recovery image. These actualities reduce the shape, shade, surface and rest of the image work. The above features that approach focuses the shade, shape and texture.RGB color is Wideband 
development rather than normal color space. CPM will give an instanced index that use the texture in this we get an image is listed below as a vector (w1, w2, w3, w4, w5, w6) it gives the predicted percentage of texture.

Although, any histogram will be in appropriate for constructing green indexes for image statistics base. But a histogram with number of range of containers will not give best boom in the computational value. Existing algorithm gives the shade of histogram with Quadratic Form $(\mathrm{QF})$ distance as same degree and the coloration histogram with Euclidean Distance its compared with their performs. But they couldn't reply properly to shifted or translated image. When a circled image is given as input, then authentic image is get back as same image because the closest healthy image. CLSFH [13] has very lesser parameter indexes and it can capture very high colorization in image.

In CLSFH we are using non-uniform quantized HSV (Hue Saturation Velocity) colour model. Calculate the mean and the same old deviation for every pixel. CLSFH helpful for measure Local Histogram and mean SD. Content Based Image Retrieval (CBIR) systems majorly based on shape of the image and the use of non-variant image moments. Moments are different types but here we are considering two moments. Those are Moment Invariants (MI) and Zernike Moments (ZM). These are moments to represent the top of the form functions of any image. Although, MI gives the non-orthogonality of the CBIR and ZM restrict their software in CBIR in terrible reconstruction. For real programs we require Faster and correct CBIR algorithms. It can be performed by using a classifier. We have deferent type of classifiers available, which includes SVM. It is a classifier and supervised getting to know the approach used for any image.

\section{A. Histogram}

To provide an explanation for a histogram we are using a bar graph. Here we are taking $\mathrm{X}$ tonal scale on one axis. On the other axis we consider pixel quantity of image. For example, the graph of a luminance histogram shows the wide variety of pixels for each brightness stage (from black to white), and whilst there are more pixels, the height at the certain luminance degree is better in histogram.

\section{B. Color Histogram}

Color histogram represents one kind styles of colors seemed and finally appears the range of pixels in every form of the colors. The 3 colors represents brightness distribution in every man or woman Red/Green/Blue color channel. Color histogram means it is combination of histogram of three planes.

\section{Edge orientation histogram}

Now for divided sub-block images calculates Edge orientation histogram. This can be do with 'Sobel' operators. The next 5 operators (vertical, horizontal, diagonals and non-directional) ought.

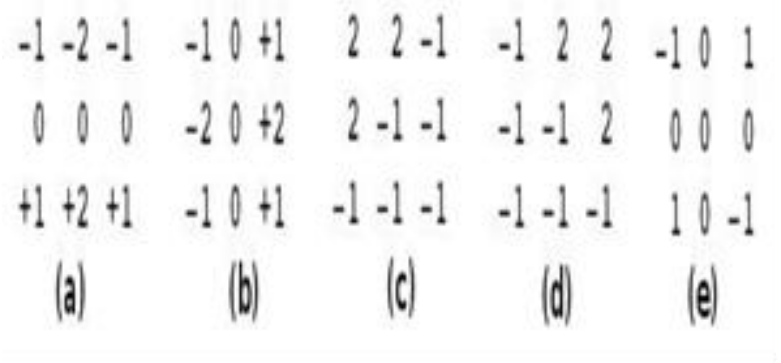

Figure 15 Orientations of Sobel Masks

Mainly depends on below parameters:

i, Scale-invariant feature transform.

ii, Shape Context.

iii, Obtained from gradient information.

iv, Complete reliance on spatial information.

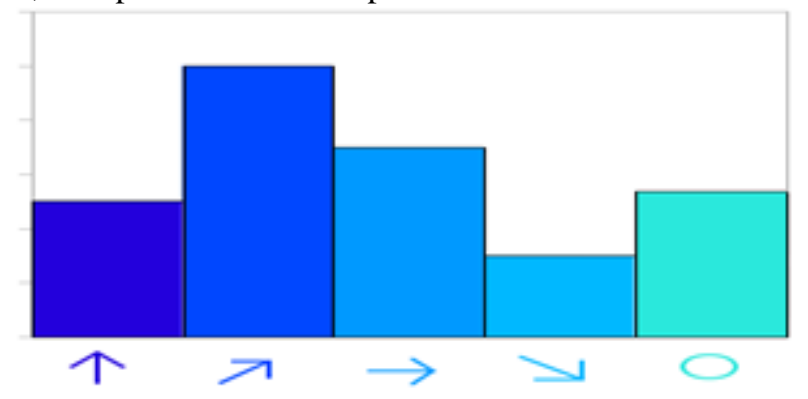

Figure 2 EOH Graph

Fig. 2 represents the image retrieval contraption by content. Set of image database could be available from that database required images may be extracted. As shown in fig. here, we are taking information as Input and given to photo to QBIR block. Totally QBIR technique is not unique approach. Because of based on image CBIR will change. Here, we are using some new techniques.

A) Relevant Feedback (Human Interaction).

B) Semantic-based image retrieval.

C) Extraction of Color.

D) Texture Feature Extraction.

E) Wavelet-Based CBIR.

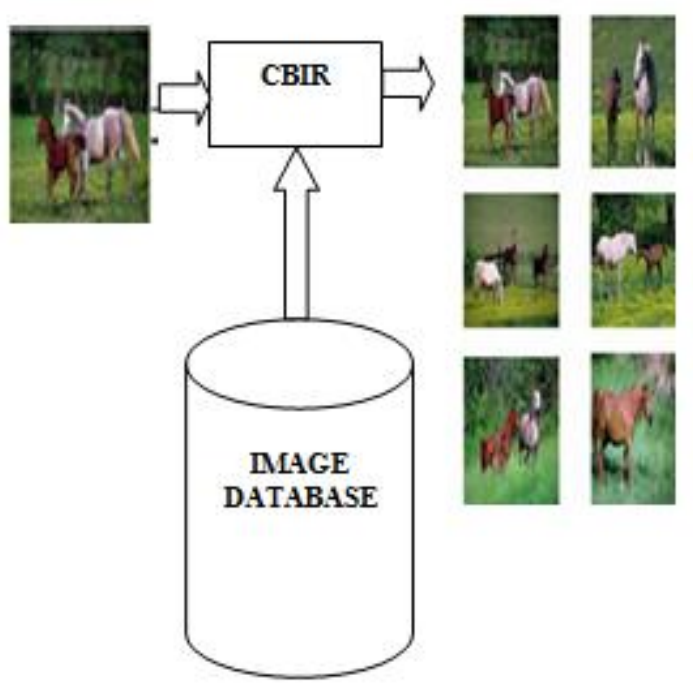

Figure 3 Basic structure of QBIR by talking reference database 


\section{PROPOSED METHOD}

\subsection{Feature Extraction}

\section{A. Color Histogram Calculation}

This project performance can be test by various quantification numbers. The color values of each block depicted as $\mathrm{C}_{\mathrm{i}(\mathrm{x} y)}(\mathrm{i} \in(0, \mathrm{n}))$, then each and every block color histogram is indicated in equation 1.

$$
H_{c_{i}(x, y)}=\operatorname{num}_{j},(j=0,1, \ldots, L-1)
$$

\section{B. EOH Measurement}

$$
\begin{gathered}
g_{R x}=\left[\begin{array}{lll}
-1 & 0 & 1 \\
-2 & 0 & 2 \\
-1 & 0 & 1
\end{array}\right] \times R, g_{G x}=\left[\begin{array}{lll}
-1 & 0 & 1 \\
-2 & 0 & 2 \\
-1 & 0 & 1
\end{array}\right] \times G, g_{B x} \\
=\left[\begin{array}{lll}
-1 & 0 & 1 \\
-2 & 0 & 2 \\
-1 & 0 & 1
\end{array}\right] \times B \\
g_{R y}=\left[\begin{array}{ccc}
1 & 2 & 1 \\
0 & 0 & 0 \\
-1 & -2 & -1
\end{array}\right] \times R, g_{G y} \\
=\left[\begin{array}{ccc}
1 & 2 & 1 \\
0 & 0 & 0 \\
-1 & -2 & -1
\end{array}\right] \times G, g_{B y} \\
=\left[\begin{array}{ccc}
1 & 2 & 1 \\
0 & 0 & 0 \\
-1 & -2 & -1
\end{array}\right] \times B
\end{gathered}
$$

Where

$g_{x x}, g_{x y}$ and $g_{y y}$ are called as dot products is observe in eq (4),(5),(6) for the vectors mentioned above.

Using the above representation, we can simply observed the MGO of $\mathrm{x} y$ is

$$
\varphi(x, y)=\frac{1}{2} \arctan \left(\frac{2 g_{x y}}{g_{x x}-g_{y y}}\right)
$$

Here, GM of x y given by

$$
\begin{aligned}
G(x, y)=\left\{\frac { 1 } { 2 } \left[\left(g_{x x}+g_{y y}\right)\right.\right. & \\
& +\left(g_{x x}-g_{y y}\right) \cos 2 \varphi \\
& \left.\left.+2 g_{x y} \sin 2 \varphi\right]\right\}^{1 / 2}
\end{aligned}
$$

Pair of orthogonal directions is given by

$$
\begin{gathered}
G_{1}(x, y)=\left\{\frac { 1 } { 2 } \left[\left(g_{x x}+g_{y y}\right)\right.\right. \\
+\left(g_{x x}-g_{y y}\right) \cos 2 \varphi \\
\left.\left.+2 g_{x y} \sin 2 \varphi\right]\right\}^{1 / 2} \\
G_{2}(x, y)=\left\{\frac { 1 } { 2 } \left[\left(g_{x x}+g_{y y}\right)\right.\right. \\
+\left(g_{x x}-g_{y y}\right) \cos 2\left(\varphi_{0}+\pi / 2\right) \\
\left.\left.+2 g_{x y} \sin 2\left(\varphi_{0}+\pi / 2\right)\right]\right\}^{1 / 2}
\end{gathered}
$$

In practical applications, we will be considering the maximum of the gradient direction. So simply, the GD is given by

$$
\varphi(x, y)= \begin{cases}\varphi_{0} & \text { ifmax }\left(G_{1}, G_{2}\right)=G_{2}(x, y) \\ \varphi_{0}+\pi / 2 & \text { if } \max \left(G_{1}, G_{2}\right)=G_{2}(x, y)\end{cases}
$$

The EOH of square depicted below

$$
H_{\varphi_{i}(x, y)}(j)=\operatorname{num}_{j},(j=0,1, \ldots, m-1)
$$

\section{Comprehensive Feature Representation}

Here we invented one algorithm i. e CFE algorithm. The step-by-step procedure for CFE is

Step1: Apply minority costume image as input to the system. Divide image into $\mathrm{n}$ sub-blocks.

Step2: Then, measure colour histogram for each subblock. Integrate them as $\mathrm{H}=[\mathrm{H}, \mathrm{H}, \ldots, \mathrm{H}]$.

Step3: Measure EOH of each sub-block. Integrates them as shown in eq (13)

$$
\mathrm{H}_{\varphi(\mathrm{x}, \mathrm{y})}=\left[\mathrm{H}_{\varphi_{1}(\mathrm{x}, \mathrm{y})}, \mathrm{H}_{\varphi_{2}(\mathrm{x}, \mathrm{y})}, \ldots . \mathrm{H}_{\varphi_{\mathrm{n}}(\mathrm{x}, \mathrm{y})}\right]
$$

Step4: Integrate all histograms derived in step2,3 i.e $\mathrm{H}=$ $\left.H=\left|H_{c(x, y)}, H_{\varphi 1(x, y)}\right|,(i \in \mid 0, \mathrm{n})\right)$

\subsection{Distance Measurement}

This project improves the results by using Canberra distance [22] [23] as a new distance metric.

$$
\begin{aligned}
D(Q, T)=\lambda \sum_{i=1}^{C S A} \frac{\left|H_{i}(Q)\right|-H_{i}(T)}{1+H_{i}(Q)+H_{i}(T)} \\
+(1 \\
\quad-\lambda) \sum_{j=1}^{C S B} \frac{\left|H_{i}(Q)\right|-H_{i}(T)}{1+H_{i}(Q)+H_{i}(T)}
\end{aligned}
$$

\section{EXECUTION RESULTS}

The proposed work as discussed above is developed using MATLAB-2016 software. As MATLAB is high level technical computing language and easy for analysis and development we selected it. In MATLAB recently there is advance features are included as GUI (Graphical User Interface). GUI helps to analyze final output effectively

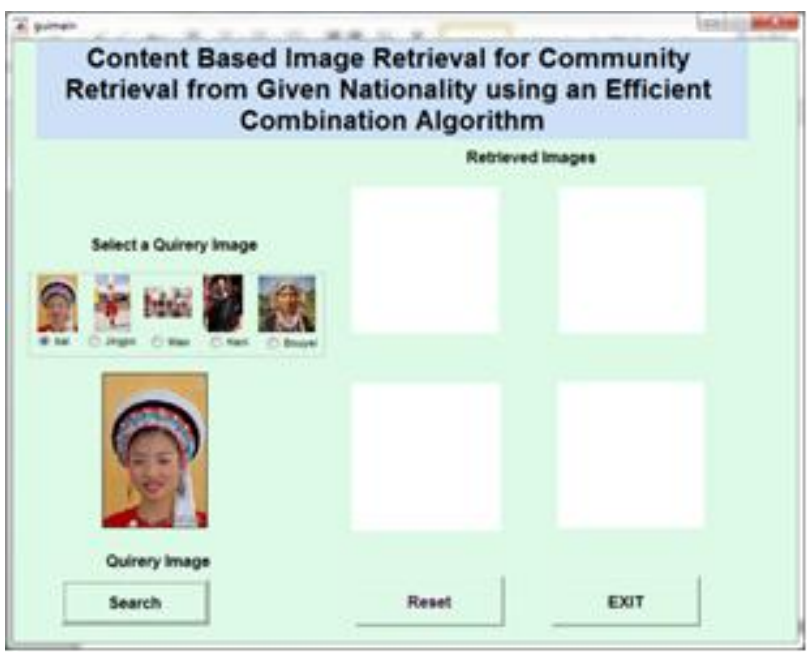

Figure 4 Query color image as input

We selected a query image from available database of bai nationality. From given image we will calculate integrated features as color histogram and edge oriented histogram features. 


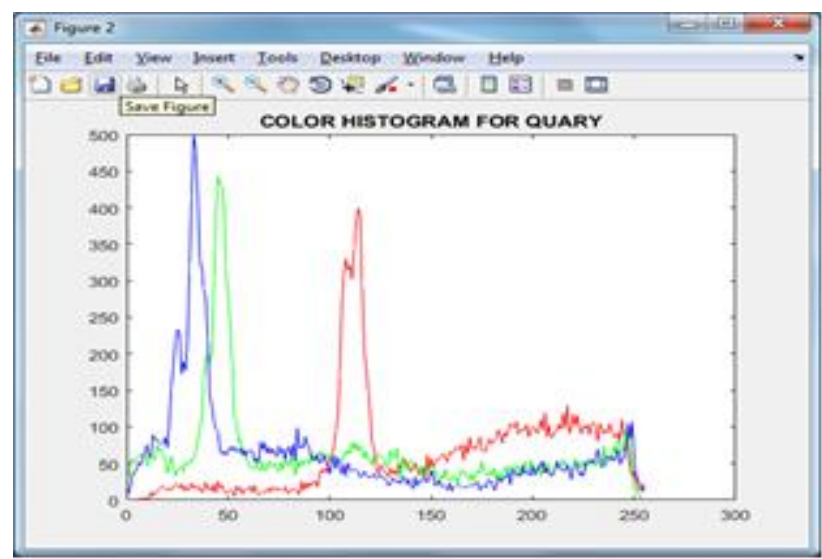

Figure 5 Color Histogram for Query image

Color histogram as shown in above diagram is combination of three histograms. R- plane histogram, Gplane histogram and B- plane histograms are combined to get final color image histogram.

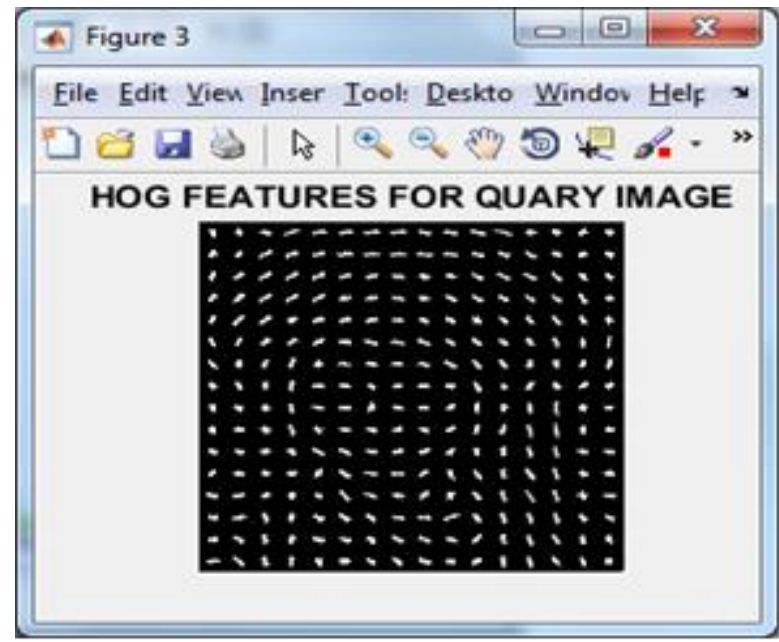

Figure 6 HOG feature for Query image

Histogram Oriented Gradient features are extracted from query image which we have to compare with database image HOG features.

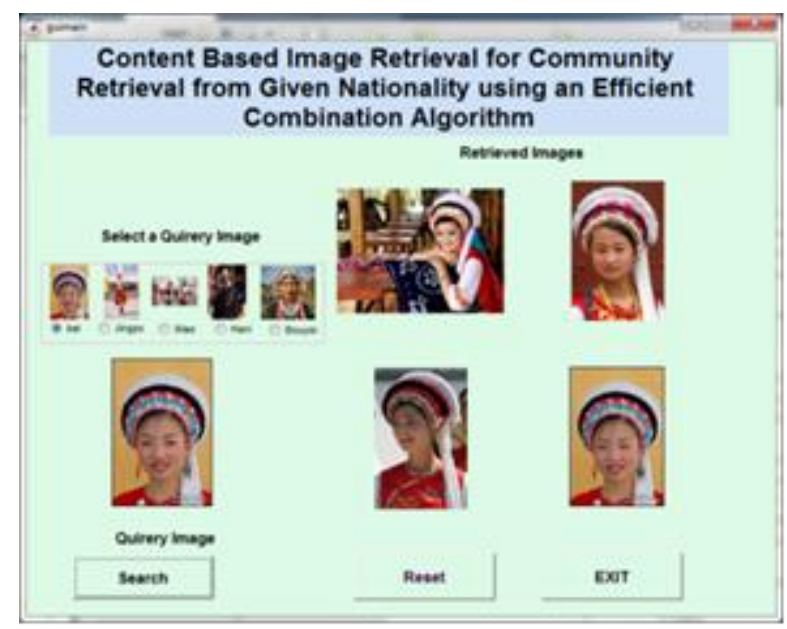

Figure 7 Retrieved Images for Query Image

Above images shows retrieved images from database for given query image which are having similar features.
Features are compared with the help of Canberra Distance [15].

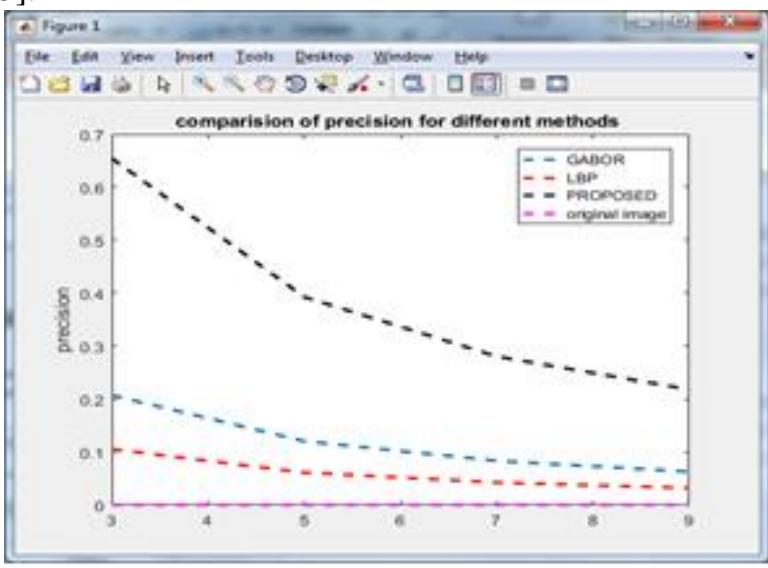

Figure 8 Precision Comparison of Existing and Proposed Work

Precision is the objective analysis parameter used for the comparison of proposed work with existing state of art techniques.

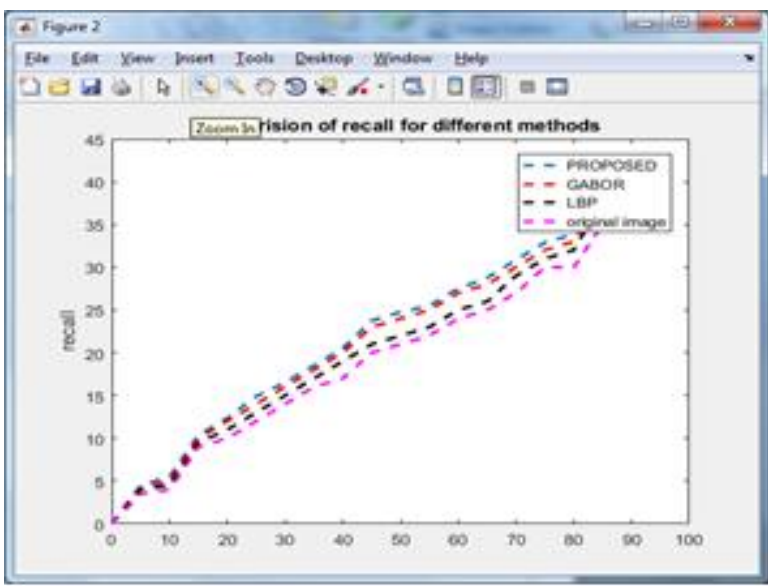

Figure 9 Recall Comparison of Existing and Proposed Work

Recall is the objective analysis parameter used for the comparison of proposed work with existing state of art techniques.

\section{CONCLUSION}

The proposed work developed and analyzed successfully by using MATLAB software based advance GUI. With the help of proposed technique we got an efficient and more accurate results compared with state of art existing techniques as GABOR filter and Local Binary Patter. By both subjective and objective analysis by precision and recall we can conclude that proposed work performs better compared to state of art methods. Here in this work we worked on local database further we can extend it for high level database as well as we can make it for global applications. Further we can extend it to salient region feature extraction for run time complexity reduction.

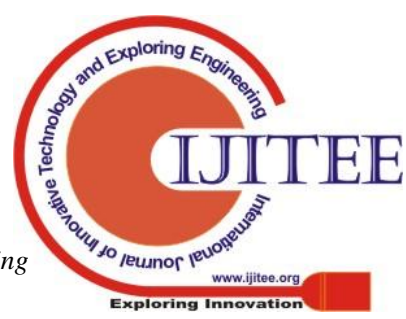




\section{REFERENCES}

1. Dana.H.Ballard and all, "Colour Indexing", IJCV-1991, Pages from 11 to 32 .

2. Markus. Orengo and all, "Similarity of Colour Images", SPIE-1995, Pages from 381 to 392.

3. Justin.Miller and all, "Comparing Images Using CCV", ACMICM-1996, Pages from 65 to 73.

4. S. Ravi Kumar and all, "Image Indexing using CC", Pages from 762 to 768, Conference on CVPR in 1997.

5. P. L. Devi, T. S. Reddy, "CBIR using FCH \& SOH", IJMETMR-2017, ISSN No:2348-4845.

6. ITS'HAK Dinstein and all, "Texture Feature for Image Classification", Pages from 610 to 621, Nov 1973, IEEE Transactions.

7. Topi.Maenpaa and all, "Multi-Resolution Gray-Scale \& Rotation Invariant Texture Classification with LBP", 0162-8828/02, July-2002, Volume 24.

8. Okson Chae and all, "Local Directional Pattern for Face Recognition", IJICIC-2012, ISSN No: 1349-4198, Pages from 2423 to 2437.

9. B. Divyavani, K. M. Reddy, "CBIR using Fusion of Colour Histogram and EOH", ISSN No: 2456-5083, IJIEMR-2017, Volume 06, Issue 12.

10. Andriew J. Newell and all, "Multiscale Histogram of oriented Gradient Descriptors for Robust Character Recognition", ICDAR-2011.

\section{AUTHORS PROFILE}

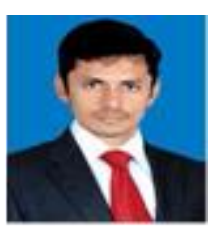

Mr. Shaik Rahamtula received B. Tech degree in the department of Electronics and Communication Engineering, from Khader Memorial College of Engineering and Technology (KMCET) (Affiliated to Jawaharlal Nehru Technological University, Hyderabad), India in 2009. Received M.Tech in ECE with the specialization Digital Electronics and Communication Engineering from QIS college of Engineering and Technology (Affiliated to Jawaharlal Nehru Technological University, Kakinada), India in 2012. Pursuing $\mathrm{PhD}$ in VELS Institute of Science Technology \& Advanced Studies (VISTAS), Chennai. His research interests in the area of Face recognition, Content Based Image Retrieval, Image Compression, Image Water Marking, Pattern Recognition, Information Retrieval and Data Mining and Wavelet Neural Network. His research work extending on medical images and working on the project titled "An Automatic segmentation of optic disc and cup region from medical images for glaucoma detection".

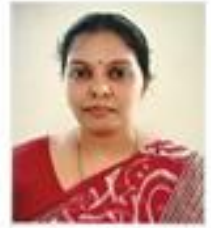

Dr. T. Jaya received the B.E. degree in Electronics and Communication Engineering from the Anna University, Chennai, India, in 2008, M.E. in Electronics and Communication Engineering (Applied Electronics) in Sathyabama University and Ph.D in Electronics and Communication Engineering in Institute of Science Technology \& Advanced Studies (VISTAS), Chennai. Currently working as Asst. Professor in Dept of ECE, VELS Institute of Science Technology \& Advanced Studies (VISTAS), Chennai. Her research interest includes Wireless Communication, Mobile Ad hoc networks and Sensor Networks Communication networks. Her research work extended with underwater wireless communication and working on the project titled "Performance Analysis on Data throughput and Bit Rate using CDMA Technique for any Underwater Acoustic Communication" 